\section{Nintedanib ist bei der idiopathischen Lungen- fibrose auch langfristig sicher}

Crestani B et al. Long-term safety and tolerability of nintedanib in patients with idiopathic pulmonary fibrosis: results from the open-label extension study, INPULSIS-ON. Lancet 2018; doi:10.1016/S2213-2600(18)30339-4

Der Angiogenese-Inhibitor Nintedanib verzögerte bei Patienten mit einer idiopathischen Lungenfibrose die Progression. Die INPULSIS-Studien ergaben eine verglichen mit Plazebo verlangsamte Reduktion der forcierten Vitalkapazität bei einem vertretbaren Nebenwirkungsprofil. In der Folgestudie INPULSIS-ON erhielten alle Teilnehmer Nintedanib. Nach 44,7 Monaten resultierten keine neuen Sicherheitsbedenken.

Der Tyrosinkinase-Inhibitor Nintedanib hemmt den Gefäßwachstumsfaktor VEGF, Fibroblastenwachstumsfaktor FGF und den Platelet-derived Wachstumsfaktor, die bei der idiopathischen Lungenfibrose pathogenetisch bedeutsam sind. INPULSIS 1 und 2 waren plazebokontrollierte Phase-3-Studien über einen Gesamtzeitraum von 52 Wochen. Die Patienten aus 24 Ländern waren $\geq 40$ Jahre alt, hatten eine forcierte Vitalkapazität (FVC) $\geq 50 \%$ und eine CO-Diffusionskapazität von $30-79 \%$. Von 807 Patienten nahmen 734 (91\%) an INPULSIS-ON teil. $59 \%$ setzten die Behandlung mit Nintedanib fort und $41 \%$ wechselten von Plazebo zu Nintedanib. Die Dosis betrug $2 \times$ täglich $150 \mathrm{mg}$. Eine Dosisanpassung auf $2 \times 100 \mathrm{mg}$ und Unterbrechungen wegen Unverträglichkeiten waren erlaubt. Primäre Endpunkte waren die langfristige Sicherheit und Verträglichkeit von Nintedanib.

Die Teilnehmer an IMPULSIS-ON waren überwiegend männlich, häufiger Weiße, durchschnittlich 67,2 Jahre alt und hatten eine mittlere FVC von 76,2\%. Die Expositionszeiten betrugen 31,5 Monate (Nintedanib in INPULSIS-ON) und 44,7 Monate (Nintedanib in INPULSIS und INPULSIS-ON). $72 \%$ der Patienten, die vor- mals Plazebo erhalten hatten, und 69\% mit kontinuierlichem Nintedanib brachen die Therapie ab. Mehr als jeder 3 . Patient setzte mindestens 1-mal die Behandlung aus oder reduzierte die Dosis. Das Nebenwirkungsprofil von Nintedanib entsprach dem aus INPULSIS. Am häufigsten waren Diarrhoen. Diese führten bei $5 \%$ (Nintedanib in INPULSIS und INPULSIS-ON) und $10 \%$ (Nintedanib in INPULSIS-ON) zum dauerhaften Therapieabbruch. In $12 \%$ und $14 \%$ der Fälle war eine Progression der Grunderkrankung Ursache für den Therapiestopp. Blutungen $(8,4 / 100$ Expositionsjahre und 6,7/ 100 Expositionsjahre) und kardiovaskuläre Ereignisse (1,3/100 Expositionsjahre und 0,7/100 Expositionsjahre) waren selten. Erhöhungen der Leberwerte über den 3-fachen Normalwert kamen bei 3\% und $7 \%$ vor.

Die FVC nahm bis Woche 192 um 327,2 $\mathrm{ml}$ ab (alle behandelten Patienten). Dies entsprach einer mittleren Reduktion um $7 \%$ und einer jährlichen Rate von - 135, 1 $\mathrm{ml}$. Männer und initial mit N-Acetylcystein behandelte Patienten waren besonders betroffen. In den Gruppen mit kontinuierlichem Nintedanib und Therapiestart in INPULSIS-ON betrugen die Exazerbationsraten 5,8/100 Personenjahre und 5,2/100 Personenjahre. 24\% und $27 \%$ der Kranken starben.

FAZIT

Die Ergebnisse aus INPULSIS bestätigten sich in der Nachfolgestudie INPULSIS-ON. Neue Sicherheitseinschränkungen und Unverträglichkeiten resultierten nicht. Die jährliche Abnahme der FVC unterschied sich nicht wesentlich von der Vorgängerstudie und unterschritt die Reduktionen der Plazebogruppe in INPULSIS. Daher gehen die Autoren auch von einer fortgesetzten Effektivität aus. Eine Datenverzerrung durch den Ausschluss von Patienten mit fortgeschrittener funktioneller Einschränkung und schweren Komorbiditäten sei möglich.

Dr. med. Susanne Krome, Melle 